\title{
Soliton Solutions for the Super mKdV and sinh-Gordon Hierarchy
}

\author{
J.F. Gomes, L.H. Ymai and A.H. Zimerman \\ Instituto de Física Teórica-UNESP \\ Rua Pamplona 145 \\ 01405-900 São Paulo, Brazil
}

\begin{abstract}
The dressing and vertex operator formalism is emploied to study the soliton solutions of the $N=1$ super $\mathrm{mKdV}$ and sinh-Gordon models. Explicit two and four vertex solutions are constructed. The relation between the soliton solutions of both models is verified.
\end{abstract}

\section{Introduction}

A systematic construction of supersymmetric integrable hierarchies within the algebraic formalism was proposed in [1]. An interesting feature of such approach is that it allows time evolutions according to both, positive and negative grades. In particular the first negative grade time evolution is always associated to the relativistic integrable model (with appropriated choice of space-time coordinates). Specific examples were given in connection with the $s l(2,1)$ super Lie algebra yielding the super $\mathrm{mKdV}$ and sinh-Gordon models. The supersymmetric sinh-Gordon was proposed in [2] by introducing a pair of Grassmann coordinates whilst the super $\mathrm{mKdV}$, involving a single Grassmann coordinate, was proposed later in [3]. By extending Hirota's method for superfields, solutions for the super mKdV was obtained [4]. The relation between the bosonic mKdV and sinh-Gordon models was observed by a number of authors [5], [6]. This fact was explained and further extended to other integrable models in [7] (e.g. Lund-Regge and Non linear Schroedinger) and in [8] the relation between soliton solutions of both models were verified explicitly. This fact was explored in [1] to show that the super mKdV and sinh-Gordon models share the same algebraic structure and henceforth belong to the same integrable hierarchy. This is verified explicitly here by comparing the soliton solutions of both models.

In this paper we employ the algebraic formalism to study the $N=1$ super $\mathrm{mKdV}$ and sinh-Gordon models by decomposing the affine $\hat{s l}(2,1)$ into half-integer graded subspaces following ref. [1]. In order to obtain a general non-trivial soliton solution from the dressing formalism and vertex operators we employ a slightly different gradation and loop automorphism from those of ref. [1].

This paper is organized as follows. In Sect. 2 we discuss the decomposition of the affine $\hat{s} l(2,1)$ super algebra into half integer graded subspaces and construct the $\mathrm{mKdV}$ and sinhGordon models. In Sect. 3 we follow refs. [9], [6] to derive the tau functions from the dressing formalism and construct the vertex operators leading to the soliton solutions for those integrable models. In the appendices we discuss the relevant subalgebra of the affine $\hat{s l}(2,1)$ and the general four vertex solution. 


\section{The super mKdV and sinh-Gordon Hierarchy}

In this section we employ the algebraic formalism to construct an integrable hierarchy containing the mKdV and sinh-Gordon supersymmetric models. Consider the $\operatorname{sl}(2,1)$ super Lie algebra with generators

$$
h_{1}=\alpha_{1} \cdot H, \quad h_{2}=\alpha_{2} \cdot H, \quad E_{ \pm \alpha_{1}}, \quad E_{ \pm \alpha_{2}}, \quad E_{ \pm\left(\alpha_{1}+\alpha_{2}\right)}
$$

where $\alpha_{1}$ and $\alpha_{2}, \alpha_{1}+\alpha_{2}$ are bosonic and fermionic roots respectively. The integrable hierarchy is defined by choosing the grading operator

$$
Q=2 d+\frac{1}{2} h_{1}
$$

where $d$ satisfy $\left[d, T_{a}^{(m)}\right]=m T_{a}^{(m)}, T_{a}^{(m)}$ denote both $E_{\alpha}^{(m)}$ or $H_{i}^{(m)}$. The hierarchy is further specified by the constant grade one element, $E=E^{(1)}$ where,

$$
E^{(2 n+1)}=h_{1}^{(n+1 / 2)}+2 h_{2}^{(n+1 / 2)}-E_{\alpha_{1}}^{(n)}-E_{-\alpha_{1}}^{(n+1)}
$$

A key ingredient to construct the desired integrable models is the judicious choice of a subalgebra $\hat{\mathcal{G}}$ of the affine $\hat{s l}(2,1)$. This is discussed in the appendix A. The grading operator $Q$ and $E$ decomposes the associated affine super Kac-Moody algebra $\hat{\mathcal{G}}=\oplus \mathcal{G}_{l}=\mathcal{K} \oplus \mathcal{M}$ where $l$ is the degree of the subspace $\mathcal{G}_{l}$ and $\mathcal{K}=\{x \in \hat{\mathcal{G}},[x, \mathcal{K}]=0\}$ denote the kernel of $E$ and $\mathcal{M}$ its complement, i.e.,

$$
\begin{array}{ll}
\mathcal{K}_{\text {Bose }}=\left\{K_{1}^{(2 n+1)}, K_{2}^{(2 n+1)}\right\}, & \mathcal{K}_{\text {Fermi }}=\left\{F_{1}^{(2 n+3 / 2)}, F_{2}^{(2 n+1 / 2)}\right\}, \\
\mathcal{M}_{\text {Bose }}=\left\{M_{1}^{(2 n+1)}, M_{2}^{(2 n)}\right\}, & \mathcal{M}_{\text {Fermi }}=\left\{G_{1}^{(2 n+1 / 2)}, G_{2}^{(2 n+3 / 2)}\right\}
\end{array}
$$

where the generators $K_{i}, M_{i}, F_{i}$ and $G_{i}$ are constructed in terms of generators of $\hat{s l}(2,1)$ in app. A. Define the Lax operator $L=\partial_{x}+E+A_{0}+A_{1 / 2}=\partial_{x}+\mathcal{A}_{x}$ where $\mathcal{A}_{x}=A_{0}+A_{1 / 2} \in$ $\mathcal{M} \bmod \hat{c}$, i.e.,

$$
A_{0}=u M_{2}^{(0)}+\eta \hat{c}, \quad A_{1 / 2}=\bar{\psi} G_{1}^{(1 / 2)}
$$

The positive hierarchy is given in terms of the zero curvature condition

$$
\left[\partial_{x}+E+A_{0}+A_{1 / 2}, \partial_{t_{n}}+D^{(n)}+D^{(n-1 / 2)}+\cdots D^{(0)}\right]=0
$$

where $D^{(i)} \in \mathcal{G}_{i}$ and can be solved recursively decomposing eqn. (2.6) grade by grade (see for instance ref. ([1]). The solution is local and the image part of the zero and one-half grade components of (2.6) yields the time evolution for the fields defined in (2.5). For $n=3$ we find the equations of motion for the $N=1$ super $\mathrm{mKdV}$, i.e.,

$$
\begin{aligned}
4 \partial_{t_{3}} \bar{\psi} & =\partial_{x}^{3} \bar{\psi}-3 u \partial_{x}(u \bar{\psi}) \\
4 \partial_{t_{3}} u & =\partial_{x}^{3} u-6 u^{2} \partial_{x} u+3 \bar{\psi} \partial_{x}\left(u \partial_{x} \bar{\psi}\right)
\end{aligned}
$$

Observe that the equation of motion for the field $\eta$ is not fixed due to ambiguity in determining $D^{(0)} \rightarrow D^{(0)}+\rho \hat{c}$. In general, for non relativistic theories, it is necessary a more 
restrictive structure given by the dressing transformations explained in the next section. Other integrable equations are obtained for different values of $n$ in similar manner. The negative hierarchy is obtained by

$$
\left[\partial_{x}+E+A_{0}+A_{1 / 2}, \partial_{t_{-m}}+D^{(-m)}+D^{(-m+1 / 2)}+\cdots D^{(-1)}+D^{(-1 / 2)}\right]=0
$$

Both, positive and negative hierarchies (2.6) were shown to be derived from the RiemannHilbert problem for the homogeneous gradation [11]. The solution for the negative hierarchy (2.8) is, in general non-local, however, the simplest member of the negative hierarchy for $m=1$ in (2.8) has a closed local solution in terms of the zero grade group element $B \in \mathcal{G}_{0}$,

$$
A_{0}=-\partial_{x} B B^{-1}, \quad A_{1 / 2}=\bar{\psi} G_{1}^{(1 / 2)}, \quad D^{(-1 / 2)}=\psi B G_{2}^{(-1 / 2)} B^{-1}, \quad D^{(-1)}=B E^{(-1)} B^{-1}
$$

where $E^{(-1)}$ is given by (2.3) for $n=-1$. According to $Q$ in (2.2) the zero grade subalgebra is generated by $\mathcal{G}_{0}=\left\{M_{2}^{(0)}, \hat{c}\right\}$, i.e.

$$
B=\exp \left(\phi M_{2}^{(0)}+\nu \hat{c}\right)
$$

The time evolution for $t_{-1}$ is obtained from (2.8) and coincides with the Leznov-Saveliev's equation [12] when we identify $\left(x, t_{-1}\right)$ with the light cone coordinates,

$$
\begin{aligned}
\partial_{t_{-1}} \partial_{x} B B^{-1} & =-\left[E, B E^{(-1)} B^{-1}\right]-\left[\bar{\psi} G_{1}^{(1 / 2)}, \psi B G_{2}^{(-1 / 2)} B^{-1}\right] \\
\partial_{t_{-1}} \bar{\psi} G_{1}^{(1 / 2)} & =\left[E, \psi B G_{2}^{(-1 / 2)} B^{-1}\right]
\end{aligned}
$$

leading in components to the $N=1$ super sinh-Gordon equations of motion,

$$
\begin{aligned}
\partial_{t_{-1}} \partial_{x} \phi & =2 \sinh 2 \phi+2 \bar{\psi} \psi \sinh \phi \\
\partial_{t_{-1}} \partial_{x} \nu & =\psi \bar{\psi} e^{\phi}+\left(1-e^{2 \phi}\right) \\
\partial_{t_{-1}} \bar{\psi} & =2 \psi \cosh \phi \\
\partial_{x} \psi & =2 \bar{\psi} \cosh \phi
\end{aligned}
$$

The above equations are invariant under supersymmetry transformation

$$
\bar{\psi}^{\prime}=\bar{\psi}+\epsilon \partial_{x} \phi, \quad \phi^{\prime}=\phi+\epsilon \bar{\psi}
$$

The zero group element $B$ defined in (2.9) when parametrized as in (2.10) establishes a correspondence between relativistic (sinh-Gordon) and non relativistic (mKdV) field variables, i.e.

$$
u=-\partial_{x} \phi, \quad \eta=-\partial_{x} \nu
$$

\section{Dressing and Soliton Solutions}

We now construct the soliton solution for both, mKdV and sinh-Gordon models from the dressing transformation generated by $\Theta_{ \pm}$which relates two solutions of the equations of 
motion written in the zero curvature representation. In particular, it relates the vacuum and the 1-soliton solutions by a gauge transformation,

$$
\mathcal{A}_{\mu}=\Theta_{ \pm} \mathcal{A}_{\mu}^{v a c} \Theta_{ \pm}^{-1}-\left(\partial_{\mu} \Theta_{ \pm}\right) \Theta_{ \pm}^{-1}
$$

where

$$
\Theta_{-}=e^{m(-1 / 2)+m(-1) \cdots} \quad \Theta_{+}=B e^{v(1 / 2)+v(1) \cdots}
$$

where $m(-i) \in \mathcal{G}_{-i}$ and $v(i) \in \mathcal{G}_{i}$. The zero curvature representation implies for pure gauge solutions:

$$
\mathcal{A}_{\mu}^{v a c}=-\partial_{\mu} T_{0} T_{0}^{-1}, \quad \mathcal{A}_{\mu}=-\partial_{\mu} T T^{-1}
$$

which leads to the following relation

$$
\Theta_{-}^{-1} \Theta_{+}=T_{0} g T_{0}^{-1}
$$

where $g \in \hat{G}$ is an arbitrary constant element of the corresponding affine group. Suppose $T_{0}$ represents the vacuum solution,

$$
T_{0}=\exp \left(-t_{n} E^{(n)}\right) \exp \left(-x E^{(1)}\right)
$$

i.e.,

$$
\mathcal{A}_{t_{n}}^{\text {vac }}=E^{(n)}, \quad \mathcal{A}_{x}^{v a c}=E^{(1)}
$$

As consequence of (3.15) with (3.20) and (3.16) we can determine $\Theta_{ \pm}$. Consider for instance eqn. (3.15) for $\mathcal{A}_{x}$ and $\Theta_{-}$. Its zero and half grade components determine the $\mathcal{M}$ components of $m(-1 / 2)$ and $m(-1)$ through

$$
A_{1 / 2}=[m(-1 / 2), E], \quad A_{0}=[m(-1), E]+\frac{1}{2}\left[m(-1 / 2), A_{1 / 2}\right]
$$

The same equation (3.15), for grades $-1 / 2$ and -1 yields respectively

$$
\begin{aligned}
\partial_{x} m(-1 / 2) & =[m(-3 / 2), E]+\frac{1}{2}[m(-1 / 2),[m(-1), E]] \\
& +\frac{1}{2}\left[m(-1), A_{1 / 2}\right]+\frac{1}{3 !}\left[m(-1 / 2),\left[m(-1 / 2), A_{1 / 2}\right]\right] \\
\partial_{x} m(-1) & =-\frac{1}{2} m(-1 / 2) \partial_{x} m(-1 / 2)+\frac{1}{2} \partial_{x} m(-1 / 2) m(-1 / 2)+[m(-2), E] \\
& +\frac{1}{2}[m(-1 / 2),[m(-3 / 2), E]]+\frac{1}{2}[m(-1),[m(-1), E]]+\frac{1}{2}\left[m(-3 / 2), A_{1 / 2}\right] \\
& +\frac{1}{3 !}[m(-1 / 2),[m(-1 / 2),[m(-1), E]]]+\frac{1}{3 !}\left[m(-1 / 2),\left[m(-1), A_{12}\right]\right] \\
& +\frac{1}{3 !}\left[m(-1),\left[m(-1 / 2), A_{1 / 2}\right]\right]+\frac{1}{4 !}\left[m(-1 / 2),\left[m(-1 / 2),\left[m(-1 / 2), A_{1 / 2}\right]\right]\right]
\end{aligned}
$$


and determines the kernel, $\mathcal{K}$, components of $m(-1 / 2)$ and $m(-1)$ (which, in principle is non local) together with the image, $\mathcal{M}$, components of $m(-3 / 2)$ and $m(-2)$. From $A_{0}$ and $A_{1 / 2}$ given by $(2.5)$ we find for the super $\mathrm{mKdV}$

$$
\begin{aligned}
m(-1 / 2) & =\alpha_{1} G_{2}^{(-1 / 2)}+\alpha_{2} F_{1}^{(-1 / 2)}, \\
m(-1) & =\beta_{1} M_{1}^{(-1)}+\beta_{2} K_{1}^{(-1)}+\beta_{3} K_{2}^{(-1)}
\end{aligned}
$$

where

$$
\begin{aligned}
& \alpha_{1}=-\frac{1}{2} \bar{\psi}, \quad \alpha_{2}=-\frac{1}{2} \chi, \quad \beta_{1}=\frac{1}{2}\left(u-\frac{1}{2} \bar{\psi} \chi\right) \\
& \beta_{2}=\frac{1}{4} \int\left(\bar{\psi} \partial_{x} \bar{\psi}-\chi \partial_{x} \chi\right) d x-\frac{1}{2} \int u^{2} d x \quad \beta_{3}=-\frac{1}{4} \int\left(\bar{\psi} \partial_{x} \bar{\psi}+\chi \partial_{x} \chi\right) d x
\end{aligned}
$$

and $\partial_{x} \chi=u \bar{\psi}$. It also leads to the eqn. for $\eta$,

$$
2 \partial_{x} \eta=u^{2}-\partial_{x} u-\bar{\psi} \partial_{x} \bar{\psi} .
$$

The full dressing transformation $\Theta_{ \pm}$is then determined by considering higher grade terms of (3.15) with (3.20) and (3.16).

From eqn. (3.18) the following $\tau$-functions are found,

$$
\begin{aligned}
\tau_{0} & =e^{\nu}=<\lambda_{0}\left|T_{0} g T_{0}^{-1}\right| \lambda_{0}> \\
\tau_{1} & =e^{\phi+\nu}=<\lambda_{1}\left|T_{0} g T_{0}^{-1}\right| \lambda_{1}> \\
\tau_{2} & =\frac{1}{2}(\bar{\psi}-\chi) e^{\nu}=<\lambda_{0}\left|G_{1}^{(1 / 2)} T_{0} g T_{0}^{-1}\right| \lambda_{0}> \\
\tau_{3} & =\frac{1}{2}(\bar{\psi}+\chi) e^{\phi+\nu}=<\lambda_{1}\left|G_{1}^{(1 / 2)} T_{0} g T_{0}^{-1}\right| \lambda_{1}>
\end{aligned}
$$

where $\lambda_{i}, i=0,1$ denote the first two fundamental weights of $\hat{s l}(2,1)$ satisfying

$$
\hat{c}\left|\lambda_{i}>=\right| \lambda_{i}>, \quad M_{2}^{(0)}\left|\lambda_{i}>=\delta_{i, 1}\right| \lambda_{i}>
$$

and are annihilated by the positive grade generators. The soliton solution is therefore given in terms of representations of the $\hat{s l}(2,1)$ affine Lie super algebra,

$$
\phi=\ln \left(\frac{\tau_{1}}{\tau_{0}}\right), \quad \bar{\psi}=\frac{\tau_{3}}{\tau_{1}}+\frac{\tau_{2}}{\tau_{0}},
$$

For the relativistic sinh-Gordon $t_{n}=t_{-1}$ whilst for the non relativistic mKdV model $t_{n}=t_{3}$, $u=-\partial_{x} \phi$ and $\eta=-\partial_{x} \nu$.

The soliton solutions are classified in terms of the constant element $g$ in (3.18) which is constructed in terms of eigenvectors of $E^{(n)}$, i.e.,

$$
\left[E^{(2 n+1)}, F_{ \pm}(\gamma)\right]= \pm 2 \gamma^{2 n+1} F_{ \pm}(\gamma)
$$

where

$$
\begin{aligned}
& F_{-}(\gamma)=\sum_{n \in Z} M_{1}^{(2 n+1)} \gamma^{-2 n-1}+\left(M_{2}^{(2 n)}-\frac{1}{2} \hat{c} \delta_{n, 0}\right) \gamma^{-2 n} \\
& F_{+}(\gamma)=\sum_{n \in Z} G_{1}^{(2 n+1 / 2)} \gamma^{-2 n}+G_{2}^{(2 n+3 / 2)} \gamma^{-2 n-1}
\end{aligned}
$$




\subsection{Two Vertex Solution}

Consider as an illustration the case where

$$
g=e^{b_{1} F_{-}\left(\gamma_{1}\right)} e^{c_{1} F_{+}\left(\gamma_{3}\right)}
$$

where $b_{1}$ and $c_{1}$ are bosonic and fermionic coefficients respectively. By virtue of (3.29) the explicit space-time dependence on the r.h.s. of (3.26) is

$$
T_{0} g T_{0}^{-1}=e^{b_{1} \rho_{1}^{+}\left(\gamma_{1}\right) F_{-}\left(\gamma_{1}\right)} e^{c_{1} \rho_{3}^{-}\left(\gamma_{3}\right) F_{+}\left(\gamma_{3}\right)}
$$

where

$$
\rho_{i}^{ \pm}=e^{ \pm\left(2 \gamma_{i} x+2 \gamma_{i}^{2 n+1} t_{2 n+1}\right)} .
$$

The $\tau$ functions (3.26) become

$$
\begin{aligned}
\tau_{0}=e^{\nu} & =1-\frac{1}{2} b_{1} \rho_{1}^{+}+b_{1} c_{1} \rho_{1}^{+} \rho_{3}^{-}<\lambda_{0}\left|F_{-}\left(\gamma_{1}\right) F_{+}\left(\gamma_{3}\right)\right| \lambda_{0}> \\
\tau_{1}=e^{\phi+\nu} & =1+\frac{1}{2} b_{1} \rho_{1}^{+}+b_{1} c_{1} \rho_{1}^{+} \rho_{3}^{-}<\lambda_{1}\left|F_{-}\left(\gamma_{1}\right) F_{+}\left(\gamma_{3}\right)\right| \lambda_{1}> \\
\tau_{2}=\frac{1}{2}(\bar{\psi}-\chi) e^{\nu} & =c_{1} \rho_{3}^{-} \gamma_{3}+b_{1} c_{1} \rho_{1}^{+} \rho_{3}^{-}<\lambda_{0}\left|G_{1}^{(1 / 2)} F_{-}\left(\gamma_{1}\right) F_{+}\left(\gamma_{3}\right)\right| \lambda_{0}>, \\
\tau_{3}=\frac{1}{2}(\bar{\psi}+\chi) e^{\phi+\nu} & =c_{1} \rho_{3}^{-} \gamma_{3}+b_{1} c_{1} \rho_{1}^{+} \rho_{3}^{-}<\lambda_{1}\left|G_{1}^{(1 / 2)} F_{-}\left(\gamma_{1}\right) F_{+}\left(\gamma_{3}\right)\right| \lambda_{1}>
\end{aligned}
$$

where the matrix elements can be evaluated from the representation theory of the affine $\hat{s l}(2,1)$ super algebra (3.26) yielding

$$
\begin{aligned}
<\lambda_{i}\left|F_{-}\left(\gamma_{1}\right) F_{+}\left(\gamma_{3}\right)\right| \lambda_{i}> & =0 \\
<\lambda_{i}\left|G_{1}^{(1 / 2)} F_{-}\left(\gamma_{1}\right) F_{+}\left(\gamma_{3}\right)\right| \lambda_{i}> & =\frac{\gamma_{3}}{2} \frac{\left(\gamma_{1}+\gamma_{3}\right)}{\left(\gamma_{1}-\gamma_{3}\right)}\left(1-2 \delta_{i, 1}\right), \quad i=0,1
\end{aligned}
$$

Since, in our formulation, the super mKdV belongs to the same hierarchy as the super sinhGordon, its solution is determined using the same vertex functions (3.30) and substituting $\gamma_{i}^{-1} t_{-1}$ by $\gamma_{i}^{3} t_{3}$ together with the change of variables $u=-\partial_{x} \phi, \eta=-\partial_{x} \nu$. The explicit space-time dependence according to the sinh-Gordon or $\mathrm{mKdV}$ are given respectively by,

$$
\rho_{i S-G}^{ \pm}=e^{ \pm\left(2 \gamma_{i} x+2 \gamma_{i}^{-1} t_{-1}\right)} \quad \rho_{i m K d V}^{ \pm}=e^{ \pm\left(2 \gamma_{i} x+2 \gamma_{i}^{3} t_{3}\right)}
$$

The corresponding two-vertex solution for the super $\mathrm{mKdV}$ is then given by

$$
\begin{aligned}
u & =-\partial_{x} \phi=-b_{1} \gamma_{1} \rho_{1}^{+}\left(\frac{1}{1+\frac{1}{2} b_{1} \rho_{1}^{+}}+\frac{1}{1-\frac{1}{2} b_{1} \rho_{1}^{+}}\right) \\
\bar{\psi} & =\frac{c_{1} \rho_{3}^{-} \gamma_{3}-b_{1} c_{1} \rho_{1}^{+} \rho_{3}^{-} \sigma_{1,3}}{1+\frac{1}{2} b_{1} \rho_{1}^{+}}+\frac{c_{1} \rho_{3}^{-} \gamma_{3}+b_{1} c_{1} \rho_{1}^{+} \rho_{3}^{-} \sigma_{1,3}}{1-\frac{1}{2} b_{1} \rho_{1}^{+}} \\
\eta & =-\partial_{x} \nu=\frac{b_{1} \gamma_{1} \rho_{1}^{+}}{1-\frac{1}{2} b_{1} \rho_{1}^{+}}, \quad \sigma_{1,3}\left(\gamma_{1}, \gamma_{3}\right)=\frac{\gamma_{3}}{2} \frac{\left(\gamma_{1}+\gamma_{3}\right)}{\left(\gamma_{1}-\gamma_{3}\right)}
\end{aligned}
$$

For the particular case where $\gamma_{1}=-\gamma_{3}=k, b_{1}=-2$ and $c_{1}=-\frac{\xi}{k}$ our solution for $u$ and $\bar{\psi}(3.37)$ coincide (after scaling $t_{3}$ ) with the one obtained in [4] by extending the bilinear approach to the supersymmetric $\mathrm{mKdV}$ equation. 


\subsection{Four Vertex Solution}

We now explicit display the general 4-vertex solution where

$$
g=e^{b_{1} F_{-}\left(\gamma_{1}\right)} e^{b_{2} F_{-}\left(\gamma_{2}\right)} e^{c_{1} F_{+}\left(\gamma_{3}\right)} e^{c_{2} F_{+}\left(\gamma_{4}\right)}
$$

where $b_{i}$ and $c_{i}, i=1,2$ are bosonic and fermionic coefficients respectively and

$$
T_{0} g T_{0}^{-1}=e^{b_{1} \rho_{1}^{+}\left(\gamma_{1}\right) F_{-}\left(\gamma_{1}\right)} e^{b_{2} \rho_{2}^{+}\left(\gamma_{2}\right) F_{-}\left(\gamma_{2}\right)} e^{c_{1} \rho_{3}^{-}\left(\gamma_{3}\right) F_{+}\left(\gamma_{3}\right)} e^{c_{2} \rho_{4}^{-}\left(\gamma_{4}\right) F_{+}\left(\gamma_{4}\right)}
$$

The $\tau$ functions in (3.26) become

$$
\begin{aligned}
\tau_{0} & =e^{\nu}=1-\frac{1}{2} b_{1} \rho_{1}^{+}-\frac{1}{2} b_{2} \rho_{2}^{+}+b_{1} b_{2} \rho_{1}^{+} \rho_{2}^{+} \alpha_{1,2} \\
& +c_{1} c_{2} \rho_{3}^{-} \rho_{4}^{-}\left(\beta_{3,4}-b_{1} \rho_{1}^{+} \delta_{1,3,4}-b_{2} \rho_{2}^{+} \delta_{2,3,4}+b_{1} b_{2} \rho_{1}^{+} \rho_{2}^{+} \theta_{1,2,3,4}\right), \\
\tau_{1} & =e^{\phi+\nu}=1+\frac{1}{2} b_{1} \rho_{1}^{+}+\frac{1}{2} b_{2} \rho_{2}^{+}+b_{1} b_{2} \rho_{1}^{+} \rho_{2}^{+} \alpha_{1,2} \\
& +c_{1} c_{2} \rho_{3}^{-} \rho_{4}^{-}\left(\beta_{3,4}+b_{1} \rho_{1}^{+} \delta_{1,3,4}+b_{2} \rho_{2}^{+} \delta_{2,3,4}+b_{1} b_{2} \rho_{1}^{+} \rho_{2}^{+} \theta_{1,2,3,4}\right), \\
\tau_{2} & =\frac{1}{2}(\bar{\psi}-\chi) e^{\nu}=c_{1} \rho_{3}^{-}\left(\gamma_{3}+b_{1} \rho_{1}^{+} \sigma_{1,3}+b_{2} \rho_{2}^{+} \sigma_{2,3}+b_{1} b_{2} \rho_{1}^{+} \rho_{2}^{+} \lambda_{1,2,3}\right) \\
& +c_{2} \rho_{4}^{-}\left(\gamma_{4}+b_{1} \rho_{1}^{+} \sigma_{1,4}+b_{2} \rho_{2}^{+} \sigma_{2,4}+b_{1} b_{2} \rho_{1}^{+} \rho_{2}^{+} \lambda_{1,2,4}\right), \\
\tau_{3} & =\frac{1}{2}(\bar{\psi}+\chi) e^{\phi+\nu}=c_{1} \rho_{3}^{-}\left(\gamma_{3}-b_{1} \rho_{1}^{+} \sigma_{1,3}-b_{2} \rho_{2}^{+} \sigma_{2,3}+b_{1} b_{2} \rho_{1}^{+} \rho_{2}^{+} \lambda_{1,2,3}\right) \\
& +c_{2} \rho_{4}^{-}\left(\gamma_{4}-b_{1} \rho_{1}^{+} \sigma_{1,4}-b_{2} \rho_{2}^{+} \sigma_{2,4}+b_{1} b_{2} \rho_{1}^{+} \rho_{2}^{+} \lambda_{1,2,4}\right) .
\end{aligned}
$$

where the coefficients are given by ${ }^{1}$

$$
\begin{aligned}
\alpha_{1,2} & =\frac{1}{4} \frac{\left(\gamma_{1}-\gamma_{2}\right)^{2}}{\left(\gamma_{1}+\gamma_{2}\right)^{2}}, \\
\beta_{3,4} & =\gamma_{3} \gamma_{4} \frac{\left(\gamma_{3}-\gamma_{4}\right)}{\left(\gamma_{3}+\gamma_{4}\right)^{2}}, \\
\delta_{j, 3,4} & =\frac{\gamma_{3} \gamma_{4}}{2} \frac{\left(\gamma_{3}-\gamma_{4}\right)}{\left(\gamma_{3}+\gamma_{4}\right)^{2}} \frac{\left(\gamma_{j}+\gamma_{3}\right)}{\left(\gamma_{j}-\gamma_{3}\right)} \frac{\left(\gamma_{j}+\gamma_{4}\right)}{\left(\gamma_{j}-\gamma_{4}\right)} \quad(j=1,2), \\
\sigma_{j, k} & =\frac{\gamma_{k}}{2} \frac{\left(\gamma_{j}+\gamma_{k}\right)}{\left(\gamma_{j}-\gamma_{k}\right)} \quad(j=1,2) \quad(k=3,4), \\
\lambda_{1,2, j} & =\frac{\gamma_{j}}{4} \frac{\left(\gamma_{1}-\gamma_{2}\right)^{2}}{\left(\gamma_{1}+\gamma_{2}\right)^{2}} \frac{\left(\gamma_{1}+\gamma_{j}\right)}{\left(\gamma_{1}-\gamma_{j}\right)} \frac{\left(\gamma_{2}+\gamma_{j}\right)}{\left(\gamma_{2}-\gamma_{j}\right)}, \quad(j=3,4),
\end{aligned}
$$

\footnotetext{
${ }^{1}$ we have used the Mathematica program of ref. [10]
} 


$$
\theta_{1,2,3,4}=\frac{\gamma_{3} \gamma_{4}}{4} \frac{\left(\gamma_{1}-\gamma_{2}\right)^{2}}{\left(\gamma_{1}+\gamma_{2}\right)^{2}} \frac{\left(\gamma_{1}+\gamma_{3}\right)}{\left(\gamma_{1}-\gamma_{3}\right)} \frac{\left(\gamma_{2}+\gamma_{3}\right)}{\left(\gamma_{2}-\gamma_{3}\right)} \frac{\left(\gamma_{3}-\gamma_{4}\right)}{\left(\gamma_{3}+\gamma_{4}\right)^{2}} \frac{\left(\gamma_{1}+\gamma_{4}\right)}{\left(\gamma_{1}-\gamma_{4}\right)} \frac{\left(\gamma_{2}+\gamma_{4}\right)}{\left(\gamma_{2}-\gamma_{4}\right)}
$$

The solution for the super sinh-Gordon is then given as

$$
\begin{aligned}
& \phi=\ln \left(\frac{1+\frac{1}{2} b_{1} \rho_{1}^{+}+\frac{1}{2} b_{2} \rho_{2}^{+}+b_{1} b_{2} \rho_{1}^{+} \rho_{2}^{+} \alpha_{1,2}}{1-\frac{1}{2} b_{1} \rho_{1}^{+}-\frac{1}{2} b_{2} \rho_{2}^{+}+b_{1} b_{2} \rho_{1}^{+} \rho_{2}^{+} \alpha_{1,2}}\right) \\
& +c_{1} c_{2} \frac{\rho_{3}^{-} \rho_{4}^{-}\left(\beta_{3,4}+b_{1} \rho_{1}^{+} \delta_{1,3,4}+b_{2} \rho_{2}^{+} \delta_{2,3,4}+b_{1} b_{2} \rho_{1}^{+} \rho_{2}^{+} \theta_{1,2,3,4}\right)}{1+\frac{1}{2} b_{1} \rho_{1}^{+}+\frac{1}{2} b_{2} \rho_{2}^{+}+b_{1} b_{2} \rho_{1}^{+} \rho_{2}^{+} \alpha_{1,2}} \\
& -c_{1} c_{2} \frac{\rho_{3}^{-} \rho_{4}^{-}\left(\beta_{3,4}-b_{1} \rho_{1}^{+} \delta_{1,3,4}-b_{2} \rho_{2}^{+} \delta_{2,3,4}+b_{1} b_{2} \rho_{1}^{+} \rho_{2}^{+} \theta_{1,2,3,4}\right)}{1-\frac{1}{2} b_{1} \rho_{1}^{+}-\frac{1}{2} b_{2} \rho_{2}^{+}+b_{1} b_{2} \rho_{1}^{+} \rho_{2}^{+} \alpha_{1,2}} \\
& \bar{\psi}=c_{1} \frac{\rho_{3}^{-}\left(\gamma_{3}-b_{1} \rho_{1}^{+} \sigma_{1,3}-b_{2} \rho_{2}^{+} \sigma_{2,3}+b_{1} b_{2} \rho_{1}^{+} \rho_{2}^{+} \lambda_{1,2,3}\right)}{1+\frac{1}{2} b_{1} \rho_{1}^{+}+\frac{1}{2} b_{2} \rho_{2}^{+}+b_{1} b_{2} \rho_{1}^{+} \rho_{2}^{+} \alpha_{1,2}} \\
& +c_{2} \frac{\rho_{4}^{-}\left(\gamma_{4}-b_{1} \rho_{1}^{+} \sigma_{1,4}-b_{2} \rho_{2}^{+} \sigma_{2,4}+b_{1} b_{2} \rho_{1}^{+} \rho_{2}^{+} \lambda_{1,2,4}\right)}{1+\frac{1}{2} b_{1} \rho_{1}^{+}+\frac{1}{2} b_{2} \rho_{2}^{+}+b_{1} b_{2} \rho_{1}^{+} \rho_{2}^{+} \alpha_{1,2}} \\
& +c_{1} \frac{\rho_{3}^{-}\left(\gamma_{3}+b_{1} \rho_{1}^{+} \sigma_{1,3}+b_{2} \rho_{2}^{+} \sigma_{2,3}+b_{1} b_{2} \rho_{1}^{+} \rho_{2}^{+} \lambda_{1,2,3}\right)}{1-\frac{1}{2} b_{1} \rho_{1}^{+}-\frac{1}{2} b_{2} \rho_{2}^{+}+b_{1} b_{2} \rho_{1}^{+} \rho_{2}^{+} \alpha_{1,2}} \\
& +c_{2} \frac{\rho_{4}^{-}\left(\gamma_{4}+b_{1} \rho_{1}^{+} \sigma_{1,4}+b_{2} \rho_{2}^{+} \sigma_{2,4}+b_{1} b_{2} \rho_{1}^{+} \rho_{2}^{+} \lambda_{1,2,4}\right)}{1-\frac{1}{2} b_{1} \rho_{1}^{+}-\frac{1}{2} b_{2} \rho_{2}^{+}+b_{1} b_{2} \rho_{1}^{+} \rho_{2}^{+} \alpha_{1,2}}, \\
& \nu=\ln \left(1-\frac{1}{2} b_{1} \rho_{1}^{+}-\frac{1}{2} b_{2} \rho_{2}^{+}+b_{1} b_{2} \rho_{1}^{+} \rho_{2}^{+} \alpha_{1,2}\right) \\
& +c_{1} c_{2} \frac{\rho_{3}^{-} \rho_{4}^{-}\left(\beta_{3,4}-b_{1} \rho_{1}^{+} \delta_{1,3,4}-b_{2} \rho_{2}^{+} \delta_{2,3,4}+b_{1} b_{2} \rho_{1}^{+} \rho_{2}^{+} \theta_{1,2,3,4}\right)}{1-\frac{1}{2} b_{1} \rho_{1}^{+}-\frac{1}{2} b_{2} \rho_{2}^{+}+b_{1} b_{2} \rho_{1}^{+} \rho_{2}^{+} \alpha_{1,2}} \text {. }
\end{aligned}
$$

where $\rho_{i}^{ \pm}=\rho_{i S-G}^{ \pm}$.

The soliton solutions for the super $\mathrm{mKdV}$ are obtained by replacing $\rho_{i}^{ \pm}=\rho_{i m K d V}^{ \pm}$in (3.36) and writing $u=-\partial_{x} \phi$ and $\eta=-\partial_{x} \nu$. We have verified that our solution for the fourvertex super $\mathrm{mKdV}$ agrees with the one found in ref. [4] when $\gamma_{1}=-\gamma_{3}=k_{1}, \gamma_{2}=-\gamma_{4}=k_{2}$, $b_{1}=b_{2}=-2$ and $c_{i}=-\frac{\xi_{i}}{k_{i}}, \quad i=1,2$ (after scaling $\left.t_{3}\right)$.

It becomes clear that the soliton solutions are classified in terms of the number and in terms of the type of vertices emploied in constructing $g$. Other integrable equations within the same hierarchy and associated to higher grade time evolution, $t_{2 n+1}$, can be constructed from the zero curvature condition (2.6) by replacing $E^{(1)}$ by $E^{(2 n+1)}$ (given in (2.3)). They all share the same soliton solutions for fields $u\left(x, t_{2 n+1}\right)=-\partial_{x} \phi, \quad \eta\left(x, t_{2 n+1}\right)=$ $-\partial_{x} \nu, \quad \bar{\psi}\left(x, t_{2 n+1}\right)$ with $\rho_{i}^{ \pm}$given by $\left.\rho_{i}^{ \pm}=e^{ \pm\left(2 \gamma_{i} x+2 \gamma_{i}^{2 n+1}\right.} t_{2 n+1}\right)$. 


\section{Acknowledgments}

We are gratefull to H. Aratyn for discussions. LHY acknowledges support from Fapesp, JFG and AHZ thank CNPq for a partial support.

\section{Appendix A}

We now discuss how to implement the relevant subalgebra of the affine $\hat{s l}(2,1)$ Kac-Moody algebra in order to construct the integrable hierarchy we are interested in. Consider the $s l(2,1)$ Lie algebra with generators

$$
\left\{h_{1}=\frac{2 \alpha_{1} \cdot H}{\alpha_{1}^{2}}, \lambda_{2} \cdot H, E_{ \pm \alpha_{1}}, E_{ \pm \alpha_{2}}, E_{ \pm\left(\alpha_{1}+\alpha_{2}\right)}\right\}
$$

where $\alpha_{1}, \alpha_{2}$ are the bosonic and fermionic simple roots respectively and $\lambda_{2}$ is the second fundamental weight. The affine $\hat{s l}(2,1)$ structure is implemented by extending each generator $T_{a} \in \operatorname{sl}(2,1)$ to $T_{a}^{(q)}$ where $\left[d, T_{a}^{(q)}\right]=q T_{a}^{(q)}$. The relevant subalgebra of the affine $\hat{s l}(2,1)$ is constructed as follows. The grade one constant element $E^{(1)}$ be given in (2.3) decomposes the affine algebra into

$$
\begin{aligned}
\mathcal{K}_{\text {Bose }} & \left.=\left\{K_{1}^{(2 n+1)}=-\left(E_{a_{1}}^{(n)}+E_{-a_{1}}^{(n+1)}\right), \quad K_{2}^{(2 n+1)}=\lambda_{2} \cdot H^{(n+1 / 2)}\right)\right\} \\
\mathcal{M}_{\text {Bose }} & =\left\{M_{1}^{(2 n+1)}=-E_{\alpha_{1}}^{(n)}+E_{-\alpha_{1}}^{(n+1)}, \quad M_{2}^{(2 n)}=h_{1}^{(n)}\right\}
\end{aligned}
$$

and the fermionic sector

$$
\begin{aligned}
\mathcal{K}_{F e r m i}=F_{1}^{(2 n+3 / 2)} & =\left(E_{\alpha_{1}+\alpha_{2}}^{(n+1 / 2)}-E_{\alpha_{2}}^{(n+1)}\right)+\left(E_{-\alpha_{1}-\alpha_{2}}^{(n+1)}-E_{-\alpha_{2}}^{(n+1 / 2)}\right) \\
F_{2}^{(2 n+1 / 2)} & =-\left(E_{\alpha_{1}+\alpha_{2}}^{(n)}-E_{\alpha_{2}}^{(n+1 / 2)}\right)+\left(E_{-\alpha_{1}-\alpha_{2}}^{(n+1 / 2)}-E_{-\alpha_{2}}^{(n)}\right) \\
\mathcal{M}_{\text {Fermi }}=G_{1}^{(2 n+1 / 2)} & =\left(E_{\alpha_{1}+\alpha_{2}}^{(n)}+E_{\alpha_{2}}^{(n+1 / 2)}\right)+\left(E_{-\alpha_{1}-\alpha_{2}}^{(n+1 / 2)}+E_{-\alpha_{2}}^{(n)}\right) \\
G_{2}^{(2 n+3 / 2)} & =-\left(E_{\alpha_{1}+\alpha_{2}}^{(n+1 / 2)}+E_{\alpha_{2}}^{(n+1)}\right)+\left(E_{-\alpha_{1}-\alpha_{2}}^{(n+1)}+E_{-\alpha_{2}}^{(n+1 / 2)}\right)
\end{aligned}
$$

The algebra is then given by the commutators

$$
\begin{array}{cll}
{\left[K_{1}^{(2 m+1)}, K_{1}^{(2 n+1)}\right]=\hat{c}(m-n) \delta_{m+n+1,0},} & {\left[K_{1}^{(2 m+1)}, K_{2}^{(2 n+1)}\right]=0,} \\
{\left[K_{1}^{(2 m+1)}, M_{1}^{(2 n+1)}\right]=-2 M_{2}^{(m+n+1)}-\hat{c}(m+n) \delta_{m+n+1,0},} & {\left[K_{2}^{(2 m+1)}, M_{1}^{(2 n+1)}\right]=0,} \\
{\left[M_{2}^{(2 m)}, K_{1}^{(2 n+1)}\right]=2 M_{1}^{(2 m+2 n+1)},} & {\left[M_{2}^{(2 m)}, K_{2}^{(2 n+1)}\right]=0,} \\
{\left[M_{2}^{(2 m)}, M_{2}^{(2 n)}\right]=\hat{c}(m-n) \delta_{m+n, 0},} & {\left[M_{2}^{(2 m)}, M_{1}^{(2 n+1)}\right]=2 K_{1}^{(2 m+2 n+1)}} \\
{\left[M_{1}^{(2 m+1)}, M_{1}^{(2 n+1)}\right]=\hat{c}(n-m) \delta_{m+n+1,0},} & {\left[K_{2}^{(2 m+1)}, K_{2}^{(2 n+1)}\right]=\hat{c}(n-m) \delta_{m+n+1}}
\end{array}
$$

and

$$
\begin{array}{rlrl}
{\left[K_{1}^{(2 m+1)}, F_{1}^{(2 n+3 / 2)}\right]} & =-F_{2}^{(2(m+n+1)+1 / 2)}, & & {\left[K_{1}^{(2 m+1)}, G_{1}^{(2 n+1 / 2)}\right]=G_{2}^{(2(m+n)+3 / 2)},} \\
{\left[K_{1}^{(2 m+1)}, F_{2}^{(2 n+1 / 2)}\right]=-F_{1}^{(2(m+n)+3 / 2)},} & & {\left[K_{1}^{(2 m+1)}, G_{2}^{(2 n+3 / 2)}\right]=G_{1}^{(2(m+n+1)+1 / 2)},}
\end{array}
$$




$$
\begin{array}{rlrl}
{\left[K_{2}^{(2 m+1)}, F_{1}^{(2 n+3 / 2)}\right]=F_{2}^{(2(m+n+1)+1 / 2)},} & & {\left[K_{2}^{(2 m+1)}, G_{1}^{(2 n+1 / 2)}\right]=G_{2}^{(2(m+n)+3 / 2)},} \\
{\left[K_{2}^{(2 m+1)}, F_{2}^{(2 n+1 / 2)}\right]=F_{1}^{(2(m+n)+3 / 2)},} & {\left[K_{2}^{(2 m+1)}, G_{2}^{(2 n+3 / 2)}\right]=G_{1}^{(2(m+n+1)+1 / 2)},} \\
{\left[M_{1}^{(2 m+1)}, F_{1}^{(2 n+3 / 2)}\right]=G_{1}^{(2(m+n+1)+1 / 2)},} & {\left[M_{1}^{(2 m+1)}, G_{1}^{(2 n+1 / 2)}\right]=-F_{1}^{(2(m+n)+3 / 2)},} \\
{\left[M_{1}^{(2 m+1)}, F_{2}^{(2 n+1 / 2)}\right]=G_{2}^{(2(m+n)+3 / 2)},} & {\left[M_{1}^{(2 m+1)}, G_{2}^{(2 n+3 / 2)}\right]=-F_{2}^{(2(m+n+1)+1 / 2)},} \\
{\left[M_{2}^{(2 m)}, F_{1}^{(2 n+3 / 2)}\right]=-G_{2}^{(2(m+n)+3 / 2)},} & {\left[M_{2}^{(2 m)}, G_{1}^{(2 n+1 / 2)}\right]=-F_{2}^{(2(m+n)+1 / 2)},} \\
{\left[M_{2}^{(2 m)}, F_{2}^{(2 n+1 / 2)}\right]=-G_{1}^{(2(m+n)+1 / 2)},} & {\left[M_{2}^{(2 m)}, G_{2}^{(2 n+3 / 2)}\right]=-F_{1}^{(2(m+n)+3 / 2)},}
\end{array}
$$

and the anticommutators

$$
\begin{aligned}
& {\left[F_{1}^{(2 m+3 / 2)}, F_{1}^{(2 n+3 / 2)}\right]_{+}=2\left(K_{2}^{(2(m+n+1)+1)}+K_{1}^{(2(m+n+1)+1)}\right),} \\
& {\left[F_{1}^{(2 m+3 / 2)}, F_{2}^{(2 n+1 / 2)}\right]_{+}=\hat{c}(2 m-2 n+1) \delta_{m+n+1,0},} \\
& {\left[F_{1}^{(2 m+3 / 2)}, G_{1}^{(2 n+1 / 2)}\right]_{+}=2 M_{2}^{(2(m+n+1))}+\hat{c}(2 m+2 n+1) \delta_{m+n+1,0},} \\
& {\left[F_{1}^{(2 m+3 / 2)}, G_{2}^{(2 n+3 / 2)}\right]_{+}=-2 M_{1}^{(2 m+2 n+3)},} \\
& {\left[F_{2}^{(2 m+1 / 2)}, F_{2}^{(2 n+1 / 2)}\right]_{+}=-2\left(K_{2}^{(2 m+2 n+1)}+K_{1}^{(2 m+2 n+1)}\right),} \\
& {\left[F_{2}^{(2 m+1 / 2)}, G_{1}^{(2 n+1 / 2)}\right]_{+}=2 M_{1}^{(2 m+2 n+1)},} \\
& {\left[F_{2}^{(2 m+1 / 2)}, G_{2}^{(2 n+3 / 2)}\right]_{+}=-2 M_{2}^{(2 m+2 n+2)}-\hat{c}(2 m+2 n+1) \delta_{m+n+1,0},} \\
& {\left[G_{1}^{(2 m+1 / 2)}, G_{1}^{(2 n+1 / 2)}\right]_{+}=2\left(K_{2}^{(2 m+2 n+1)}-K_{1}^{(2 m+2 n+1)}\right),} \\
& {\left[G_{1}^{(2 m+1 / 2)}, G_{2}^{(2 n+3 / 2)}\right]_{+}=\hat{c}(2 m-2 n-1) \delta_{m+n+1,0},} \\
& {\left[G_{2}^{(2 m+3 / 2)}, G_{2}^{(2 n+3 / 2)}\right]_{+}=-2\left(K_{2}^{(2 m+2 n+3)}-K_{1}^{(2 m+2 n+3)}\right)}
\end{aligned}
$$

where the index $l$ in $K_{i}^{(l)}, M_{i}^{(l)}, F_{i}^{(l)}$ and $G_{i}^{(l)}$ denote their grade with respect to $Q$ given in $(2.2)$.

\section{References}

[1] H. Aratyn, J.F. Gomes and A.H. Zimerman, Nucl. Phys. B676 (2004) 537, hepth/0309099

[2] P. Di Vecchia and S. Ferrara, Nucl. Phys. B130 (1977) 93; M. Chaichian and P. Kulish, Phys. Lett. 78B (1978) 413

[3] P. Mathieu, J. Math. Phys. 29 (1988) 2499; L. Yamanaka and R. Sasaki, Prog. Theor. Phys. 79(1988), 1167

[4] Q.P. Liu, Xing-Biao Hu and Meng-Xia Zhang, Nonlinearity 18 (2005) 1597

[5] A. Chodos, Phys. Rev. D 212818 (1980); C. A. Tracy and H. Widow, Commun. Math. Phys. 1791 (1996)[solv-int/9506006]; J. Dorfmeister, H. Gradl and J. Szmigielski, Acta Applicandae Math. 53 (1998) 1; D. Fioravanti and M. Stanishkov, Nucl. Phys. B 591, 685 (2000) [hep-th/0005158]; 
[6] L.A. Ferreira, J.L. Miramontes and J. Sanches Guillen, J.Math.Phys.38,(1997),882, hepth/9606066

[7] H. Aratyn, L.A. Ferreira, J.F. Gomes and A.H. Zimerman, J. Physics A33 (2000) L331, nlin.si/0007002

[8] I. Cabrera-Carnero, J.F. Gomes, E.P. Gueuvoghlanian, G.M. Sotkov and A.H. Zimerman, Proc. of the 7th International Wigner Symposium (Wigsym 7), College Park, Maryland,(2001), hep-th/0109117

[9] D. Olive,, N. Turok and J. Underwood, Nucl. Phys. B409 (1993) 509

[10] A.G. Bueno, L.A. Ferreira and A. V. Razumov, Nucl. Phys. B626 (2002) 463, hepth/0105078

[11] H. Aratyn, J.F. Gomes and A.H. Zimerman,J. Geom. Phys. 46 (2003) 21, hepth/0107056

[12] A.N Leznov and M.V. Saveliev, "Group-Theoretical Method for Integration of Nonlinear Dynamical Systems" Springer Verlag (1992)

[13] H. Aratyn, G.M. de Castro, J.F. Gomes, M.B. Silka and A.H. Zimerman J. Physics A38 (2005) 9341, hep-th/0508008 